\title{
PROCEDURAL ERRORS IN ENDODONTIC TREATMENT: ANALYSIS OF PRECLINICAL STEP-BACK PREPARATION PERFORMED ON TRAINING BLOCKS
}

\author{
Anna Lehmann, Kacper Nijakowski, Wojciech Eliasz, Anna Surdacka \\ Department of Conservative Dentistry and Endodontics, Poznan University of Medical Sciences, Poznan, Poland
}

\begin{abstract}
INTRODUCTION: Step-back technique is a basic method used for root canal preparation. In dental school curricula, this technique is taught in a preclinical environment, and it is regarded as safe and technically easy to perform, usually with low-risk of complications.

ОвJECTIVEs: The study aims to determine the incidence of different types of procedural errors during root canal preparation by second-year dental students using step-back technique performed on training blocks with $10 \%$ and $20 \%$ curvature.

MATERIAL AND METHODs: Study material consisted of 160 endodontic training blocks, which were prepared manually by eighty second-year dental students using step-back technique. The blocks were assessed by two teachers who specialized in conservative dentistry and endodontics. The occurrence of the following errors was evaluated: working length loss, improper debridement, alteration of original root canal shape, apical over-instrumentation, instrument separation, root canal blockage with dentine chips, apical transportation or perforation in root canal wall, and ledge formation within root canal wall.

RESULTs: Percentage of error-free blocks with $10 \%$ curvature was significantly higher than the percentage of errorfree blocks with $20 \%$ curvature ( $34 \%$ and $4 \%$, respectively). Improper debridement was the most frequently observed error in both groups ( $42 \%$ and $94 \%$, respectively).

Conclusions: Our findings indicate a gap in the correct method of imparting knowledge to dental students regarding root canal preparation techniques. This disparity can be used constructively to create better training programs and more effective modules for dental students to minimize their margin of error.
\end{abstract}

KEY WORDS: root canal preparation, step-back technique, iatrogenic errors, training techniques, undergraduate dental education.

J Stoma 2019; 72, 6: 256-262

DOI: https://doi.org/10.5114/jos.2019.93845

\section{INTRODUCTION}

Endodontics constitutes one of the youngest and the most challenging fields of modern dentistry. Thanks to new and improved instruments and materials, dynamic development within this field can be observed; however, endodontic treatment still presents a significant challenge, not only for students, but also for experienced clinicians. The so-called "pyramid of success" in endodontics makes us aware that even small errors or mishaps during early stages of treatment can unfavorably influence the final result. Improper access cavity preparation

\section{JOURNAL OF STOMATOLOGY CZASOPISMO STOMATOLOGICZNE

ADDRESS FOR CORRESPONDENCE: Kacper Nijakowski, Department of Conservative Dentistry and Endodontics, Poznan University of Medical Sciences, 70 Bukowska Str., 60-812 Poznan, Poland, e-mail: kacpernijakowski@ump.edu.pl

ReceIved: 06.01.2020 • ACCEPTED: 24.02.2020 • Published: 10.03.2020 
is an example of such an error, as it may lead to missing a root canal during the cleaning and shaping stage.

Preclinical education has a significant influence on the process of undergraduate dental education. The syllabus of preclinical endodontic courses should be adapted as per the students' cognitive skills, but it should also take into account dynamic developments within the field of dentistry. A perfect simulation model should allow a student to practice all available modern methods to meet the requirements of contemporary endodontics. It seems particularly important that students should be aware of consequences resulting from their seemingly insignificant actions, to help them understand the issue and receive clear instructions from their instructors, which may prevent them from committing iatrogenic mistakes.

The step-back technique, which is commonly used and taught in dental schools, is a secure method that does not require a high level of technical skills or expensive instruments. This method enables a successful preparation of root canal with traditional instruments, NiTi files, or instruments with modified non-cutting tips. In addition, applying the principle of pre-curving an instrument to adapt it to a root canal curvature is very important. It appears that manual methods for a root canal preparation are popular among students because of the belief that rotary instrumentation is reserved for more experienced clinicians, due to the highrisk of tooth structure loss or instrument separation. While this may not be entirely accurate, it is important to note that becoming familiar with and using hand instrumentation methods at the beginning and during later stages of clinical practice is what facilitates the use of engine-driven instruments at a future stage.

Natural teeth, which are used to date in preclinical education, play an essential role. The tooth anatomy, structure of enamel and dentine, and configuration of root canals are variable features, and students should have an opportunity to learn and experience as many of this variations as possible. On the other hand, the use of transparent training blocks instead of real teeth permits to understand the behavior of instrument within the root canal, the movement of cut material, and the dynamics of irrigating fluid. These blocks are educationally important, as they allow the students to comprehend various clinical situations that are likely to occur in a professional environment.

Even though preclinical education provides an insight into root canal preparation, the effects of preparation are not always error-free. Working length (WL) loss is one of the most frequently observed errors during chemo-mechanical root canal preparation. It frequently occurs when WL is recklessly calculated, if the root canal is blocked with dentine chips due to improper irrigation, or if a ledge is formed within the root canal wall. Perforation or instrument separation are less often observed errors [19].

Loss of WL is relatively easy to recognize in clinical practice in many ways, e.g. after taking an X-ray with the instrument inside the canal. Other complications such as apical over-instrumentation, ledge formation in root canal wall, or incomplete debridement of root canal may go unnoticed by the clinician. Patient's subjective symptoms or development of inflammatory lesion in the periapical area may indicate that an error has occurred. The facility to track the instrument during its course inside root canal (via radiographic aids) has allowed to eliminate most of endodontic complications.

The awareness of possible errors during treatment is an integral part of preclinical endodontic education. In the literature, there is no answer to the question concerning the most effective ways of teaching endodontics in the preclinical setting. Furthermore, there are no clear guidelines on the use of proper instruments and methodologies. The paper constitutes an analysis of frequent mistakes observed during root canal preparation with the use of the step-back technique in preclinical endodontic courses. Information obtained may help to develop better guidelines and procedures in educating future dentists.

\section{OBJECTIVES}

The aim of the study was to determine the incidence of different types of procedural errors during root canal preparation by second-year dental students using the step-back technique performed on training blocks with $10 \%$ and $20 \%$ curvature.

\section{MATERIAL AND METHODS}

According to KB-356/18 confirmation obtained from the Poznan University of Medical Sciences Bioethics Committee, this scientific research was not a medical experiment. The study material consisted of 160 endodontic training blocks (Frasaco GmbH, Tettnang, Germany) prepared manually with the use of the step-back technique. These blocks were prepared by eighty second-year dentistry students during compulsory preclinical endodontics course at the Poznan University of Medical Sciences. Each student received one block with $10 \%$ curvature and one block with 20\% curvature. Apical diameter of both canals was $0.15 \mathrm{~mm}$, with $4-6 \%$ taper.

The blocks were mounted on phantom heads and covered with non-transparent tape, so that the student could not see the inside of root canal. New stainless-steel K-files size 15-45, and H-file size 30 (Poldent, Warsaw, Poland) were used for root canal preparation. Water and isopropyl alcohol were used for irrigation, as alcohol could dissolve the artificial colored substance (simulating pulp) within the root canal. Endogel (Chema-Elektromet, Rzeszów, Poland) was used as a lubricant. Working length (WL) was determined at $18 \mathrm{~mm}$, and $0.5 \mathrm{~mm}$ before apical foramen. Root canal preparation in the following sequence of $\mathrm{K}$-files was indicated as follows: 
\#15 WL $18 \mathrm{~mm}$, \#20 WL $18 \mathrm{~mm}$, \#15 WL $18 \mathrm{~mm}$, \#25 WL $18 \mathrm{~mm}$, \#15 WL $18 \mathrm{~mm}$, \#30 WL $18 \mathrm{~mm}$ (master apical file, MAF), \#35 WL $17 \mathrm{~mm}$, \#30 WL $18 \mathrm{~mm}$, \#40 WL $16 \mathrm{~mm}$, \#30 WL $18 \mathrm{~mm}$, \#45 WL $15 \mathrm{~mm}$, \#30 WL $18 \mathrm{~mm}$, and at the end H-file \#30 WL $18 \mathrm{~mm}$. Root canal was irrigated after each instrument using an endodontic needle (15 $\mathrm{ml}$ of liquid per canal). Students were informed that the needle should be inserted into the canal without any wedging and that the irrigant should not escape through the apex.

No time limit was given to the students. Once the preparation was completed, the tape was removed and complications analyzed. The evaluation of prepared blocks was performed by two teachers who specialized in conservative dentistry and endodontics. The following complications were observed: A - loss of WL (more than $1 \mathrm{~mm}$ ), B - improper debridement (remains of the colored wax), C - alteration of root canal shape, D - apical over-instrumentation, $\mathrm{E}$ - instrument separation within a root canal, F - root canal blockage by "dentine" chips, $\mathrm{G}$ - apical transportation or root canal wall perforation, $\mathrm{H}$ - ledge formation within a root canal wall. The complications are presented schematically in Figure 1.
The data was represented by the percentage values of cases, in which a given error occurred. The differences between the frequency of errors in the $10 \%$ curvature block and the $20 \%$ curvature block were statistically analyzed using the McNemar's test. The calculations were performed using the Statistica 13.1 software (StatSoft, Cracow, Poland). The significance level was estimated at $\alpha=0.05$.

\section{RESULTS}

The results of statistical analysis are presented in Table 1. The percentage of error-free blocks with $10 \%$ curvature was significantly higher than the percentage of error-free blocks with 20\% curvature (34\% and $4 \%$, respectively). Therefore, at least one error was found in 53 out of 80 blocks with $10 \%$ curvature. In the $10 \%$ curvature blocks, an improper debridement was the most frequently observed error, while the second most frequently observed complication was apical over-instrumentation. Alteration of root canal shape occurred relatively often (17\% of all root canals), followed by WL loss and root canal blockage with dentin chips

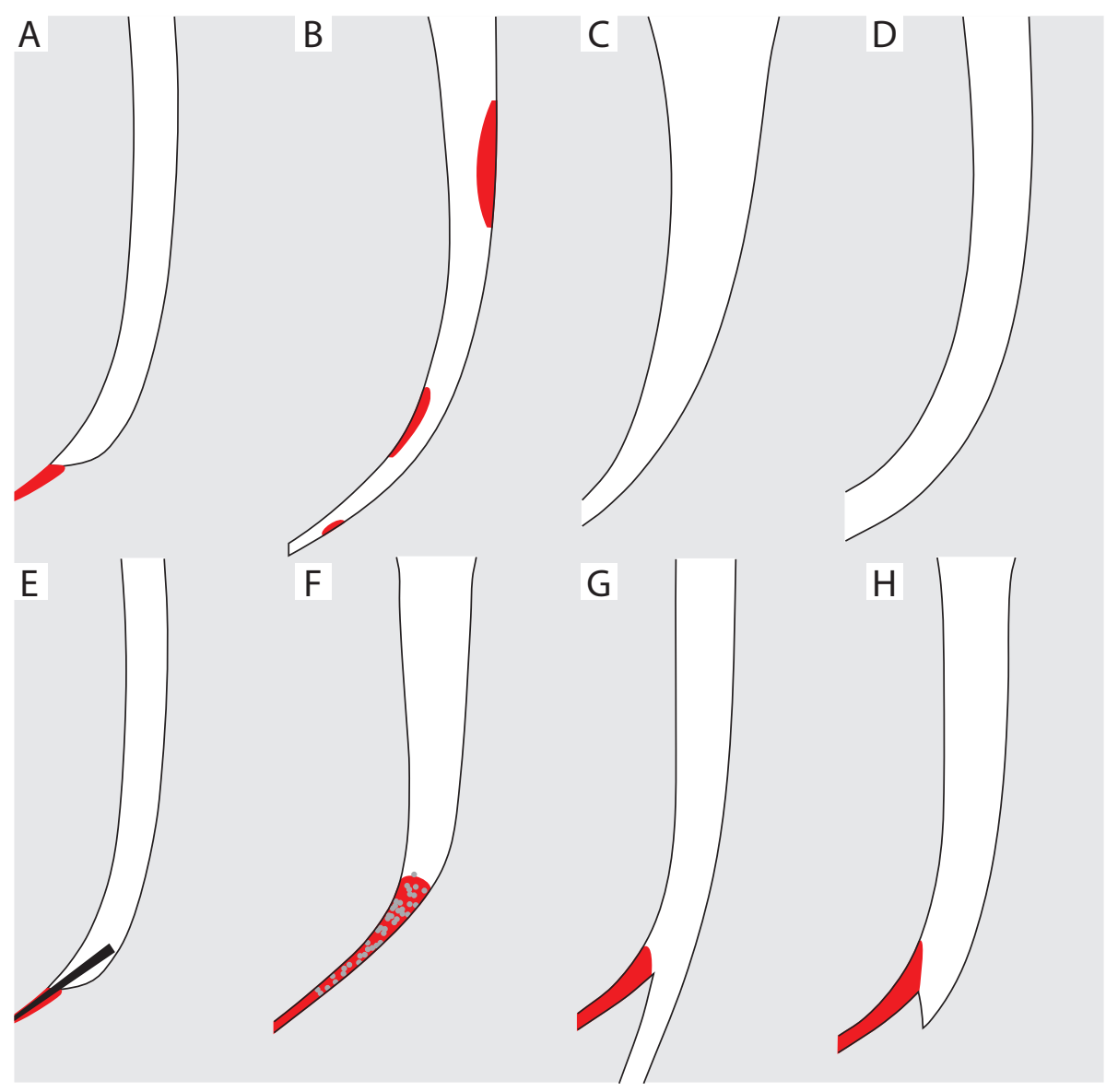

FIGURE 1. Complications during root canal preparation: A) working length loss; B) improper debridement; C) alteration of original root canal shape; D) apical over-instrumentation; E) instrument separation; F) root canal blockage with dentine chips; $\mathbf{G}$ ) root canal wall perforation; $\mathrm{H}$ ) ledge formation 
TABLE 1. The percentage of errors occurring during root canal preparation with the step-back technique in training blocks of $10 \%$ and $20 \%$ curvature

\begin{tabular}{|c|c|c|c|c|}
\hline \multirow[b]{2}{*}{ Parameter } & \multicolumn{3}{|c|}{ Training blocks (\%) } & \multirow[b]{2}{*}{$p$ value } \\
\hline & $N=160$ & $\begin{array}{c}10 \% \text { curvature } \\
n=80\end{array}$ & $\begin{array}{c}20 \% \text { curvature } \\
\quad n=80\end{array}$ & \\
\hline Improper debridement & 69 & 42 & 94 & $<0.0001^{* * *}$ \\
\hline Working length loss & 27 & 11 & 42 & $<0.0001^{* * *}$ \\
\hline Ledge & 23 & 2 & 43 & $<0.0001^{* * *}$ \\
\hline Root canal blockage with "dentine" chips & 21 & 11 & 30 & $0.0028^{* *}$ \\
\hline Alteration to the original root canal shape & 19 & 17 & 21 & 0.5176 \\
\hline Apical over-instrumentation & 18 & 23 & 12 & 0.0665 \\
\hline Apical transportation & 4 & 0 & 9 & $0.0057^{* *}$ \\
\hline Root canal wall perforation & 4 & 0 & 7 & $0.0154^{*}$ \\
\hline Instrument separation & 2 & 0 & 4 & 0.0690 \\
\hline
\end{tabular}

(11\% for each). Ledge formation was observed in only 2 blocks. Perforation of root canal was not noted in any of the blocks.

In the $20 \%$ curvature blocks, a significantly higher percentage of complications was observed. Only 3 blocks were prepared correctly, i.e. without any noticeable errors. Improper debridement was the most frequently observed complication, followed by ledge formation and WL loss (43\% and 42\%, respectively). Root canal blockage with "dentine" chips was found in as many as $30 \%$ of cases. A total of 6 cases of root perforation were observed, which were related to apical transportation. Instrument separation was found in 3 different blocks.

\section{DISCUSSION}

Significant differences in the quality of prepared blocks show that the risk of error becomes higher as the curvature of root canal increases. Teaching endodontics with the use of blocks with $0 \%$ and $10 \%$ curvature is relatively easy; however, when a student has to face a real clinical situation, this level of practice may prove insufficient. It should be noted that root canals in natural teeth are, in many cases, much more curved. Therefore, practicing the step-back technique with a $20 \%$ curvature block seems an appropriate method of instruction. Statistics show that $80-90 \%$ of natural root canals are curved, out of which $70 \%$ exhibit moderate to severe curvature [21]. Nowadays, it is generally accepted that the step-back technique enables clinicians to overcome procedural errors only in root canals, which are slightly or moderately curved, as severely curved canals are more difficult to navigate with this technique [7].

The most critical objective of root canal treatment is to minimize the number of microorganisms and debris in the root canal system and infected pulp tissue.
Locating the apical constriction in clinical situations is difficult. It is impossible to clinically locate the minor foramen with certainty, mainly because of its position and topography. The cemento-dentinal junction has also been indicated as the proper location for WL establishing, as it constitutes the point at which the pulp and periodontal tissues communicate. The location of the cemento-dentinal junction is widely accepted at 0.50-0.75 $\mathrm{mm}$ coronal to the apical foramen, but with regards to the apical constriction, it is impossible to clinically determine the exact location of the cemento-dentinal junction [18].

The establishment of WL is not sufficient, as it requires to be observed throughout procedures such as root canal preparation and obturation. Continuous monitoring of WL is crucial during whole process of treatment, especially in curved canals. The failure to adjust WL may have adverse effects. The study showed that WL loss occurred in $27 \%$ of all the blocks. Statistically significant differences between root canals with $10 \%$ and $20 \%$ curvatures seem to suggest that the difficulty of preparation increases with the degree of curvature. The method of pre-curving stainless steel instruments to adapt better to curved canals proved incredibly difficult for the students. Further, lack of visibility (the blocks were covered with non-transparent tape) also negatively influenced the errors concerning the maintenance of WL.

As suggested, endodontic treatment aims to reduce the bacterial count inside the root canal space. This is why infected tissue and root canal dentine need be removed carefully. The grade of root canal debridement was also analyzed in the study; improper debridement indicated by residual deposits of colored wax inside the training block canal was the most frequently observed complication in both groups. These results show that the students are not able to properly debride the root canal using the step-back technique. Remains of wax were observed 
in $94 \%$ of the blocks with $20 \%$ curvature. Even root canal irrigation with the use of isopropyl alcohol was not able to remove the wax effectively (isopropyl alcohol may dissolve colored wax inside the root canal; it imitates sodium hypochlorite in its tissue dissolution ability).

The study showed that WL loss occurred in $20 \%$ of all the blocks due to the blockage of apical part of the root canal with the wax material. It may also be attributed to the technique used; blockage occurred most frequently in canals, which were prepared using the step-back technique and canals with anti-curvature and circumferential filing $[2,14]$. These errors occurred least frequently when the balanced-force technique was used. Canal blockage can be avoided, if a proper instrumentation sequence is followed and copious irrigation is maintained during preparation. Moreover, the use of NiTi rotary instruments may also help to prevent this complication, as their cross-sectional design promotes coronal debris removal during clockwise rotation.

Ledge formation in the root canal wall is another filing error that frequently occurs during root canal preparation. This error may be described as a deviation from the original path of the root canal, without communication with the periodontal ligament. It causes working length loss, hinders complete preparation and disinfection, and makes root canal obturation more difficult. The incidence of ledge formation during root canal preparation is estimated at 6-60\% [10]. Numerous studies conducted with dental students as the study group showed that the percentage of prepared root canals, in which a ledge was present was much higher among the student-prepared canals than the percentage of root canals prepared by experienced clinicians ( $52 \%$ and $41 \%$, respectively $[6,13,14]$. It has also been concluded that the percentage of complications was higher in root canals in curvature exceeding 20\%. Our study confirmed this observation. The percentage of preparations with ledges was low in the blocks with $10 \%$ curvature (only $2 \%$ of cases). As the curvature increased, so did the incidence of ledges, eventually reaching a percentage of $43 \%$ in all the blocks. The difference between the groups was statistically significant.

Ledge formation is most commonly attributed to the use of inappropriate sizes (too large) of stainless-steel instruments and the use of excessive force during preparation. Incomplete preparation and debridement of the access cavity as well as endodontic treatment performed with the access gained from the mesial side, result in the loss of control over the instrument, which leads to difficulties in reaching the apical portion of root canal. Furthermore, the introduction of instrument at an incorrect angle, an improper assessment of root canal curvature, an improper working length determination, and a non-pre-curved stainless-steel instruments may all lead to ledge formation. Improper irrigation and inappropriate instrument sequence (particularly without the use of lubricants) additionally lead to root canal blockage, which in turn may cause ledge formation. It is believed that root canal blockage and ledge formation in many cases occur concurrently [10]. Overuse of chelators is another factor that contributes to ledge formation. Such substances excessively soften the dentine and predisposing it to the influence of files. All the above reasons may also be associated with incorrect manipulation of inexperienced students in preclinical endodontic classes.

An adequately prepared root canal should have tapered and uniformly smoothed walls. Unfortunately, a significant percentage of the blocks prepared by the students showed alterations in the original path of root canal; it was observed in total of $19 \%$ of blocks (17 blocks with $10 \%$ curvature, 21 blocks with $20 \%$ curvature). The taper of the walls was found to be increased significantly, mainly due to the use of excessive force by the student, particularly while working with $\mathrm{H}$ files. In the clinical environment, this may lead to the canal weakening at the point of internal curvature (the socalled "canal straightening error") and eventual perforation of root wall [20].

Apical over-instrumentation is a dangerous complication that occurs due to a change in the original path of root canals. In such cases, the shape of apical foramen is similar to a teardrop or an upside-down hourglass. Such shapes make it more difficult to seal the root canal, which in turn leads to impaired healing, development of inflammatory periapical lesions, and post-operative pain. There are two mechanisms that are responsible for apical over-preparation: a change in WL during the preparation of curved root canal, and the straightening force of instrument inside the canal, which grows with an increase in the curvature of root canal and the stiffness of instrument $[5,9]$. The study showed that the students tended to over-prepare the apical foramen more frequently in the blocks with $10 \%$ curvature than in the blocks with $20 \%$ curvature ( $23 \%$ and $12 \%$, respectively). This was because only a small number of operators were able to reach the apical foramen in the canal that was more curved, in which WL loss was observed more frequently. According to You et al. [25], a significant decrease in the risk of apical over-preparation can be achieved with the use of reciprocal engine-driven preparation.

Improper management of root canal preparation may also lead to a complete alteration of the morphology and topography of root canal due to apical transportation or lateral wall perforation. As opposed to perforation, an apical transportation is diagnosed relatively rarely $[3,22]$. The change in the location of apical foramen promotes residues of infected pulp in the root canal, which leads to pain and development of periapical lesions. The risk of apical transportation is believed to increase with an expansion in the curvature of root canal, which was confirmed in our and other studies [3, 16]. A variety of factors may influence the occurrence of this error. The original position of the apical foramen may be preserved if the instrument is pre-curved to the root 
canal curvature and an appropriate instrumentation technique is used. Many authors consider engine-driven instrumentation as better than hand instrumentation, as suggested by Abu-Tahun et al. [1]. They showed that the students who used hand instruments to prepare a root canal encountered significantly more difficulties than their peers who worked with rotary instruments (such as ProTaper) using a crown-down technique. Moreover, the students in the rotary instrumentation group were able to prepare the root canal in a much faster and safer way.

Lateral wall perforation has a significantly negative influence on the prognosis and, unfortunately, may eventually lead to extraction. Perforations located in the apical third of the root canal are particularly dangerous. During the step-back technique, perforations often result due to operator's excessive force and absence of pre-curving of instruments before their introduction into the root canal. The perforations in our study occurred much more frequently in the blocks with $20 \%$ curvature than in the blocks with $10 \%$ curvature (7\% and $0 \%$, respectively). A similar result was reported in a study by Kfir et al. [12], where perforation occurred in $7 \%$ of cases undergoing endodontic treatment performed by students.

Instrument separation was the least frequently observed error in our study. It occurred in only 3 out of 160 cases. Main reasons for instrument separation included improper preparation of access cavity, lack of knowledge concerning root canal anatomy, incorrect sequence of instruments used, improper debridement of the instruments before their reintroduction into the root canal, small volume of irrigant used, excessive forces exerted during instrumentation, faults resulting from the physical properties of the alloys, and manufacturer-related defects, which occurred very rarely $[15,17]$.

The analysis of errors made by students during root canal preparation with the step-back technique allowed to formulate some conclusions. It can be stated that the majority of errors could be avoided if the student was able to diagnose the first signs of impending complication. Even though the step-back procedure is considered a simple technique, students often made mistakes in following the sequence of instruments. The necessity to pre-curve the instruments also had a negative effect on the quality of WL determination. As already mentioned, academic teachers support the view that rotary instrumentation should be performed only by experienced clinicians due to a high-risk of tooth structure loss or instrument separation. However, numerous studies conducted among students have shown that rotary methods can be taught and skills acquired much faster without a high-risk of the discussed complications [8, 11, 23, 24]. Ribeiro et al. [19] interestingly described the level of effectiveness of endodontic treatment provided by students, where frequency of acceptable technical quality of root fillings was $48 \%$. Perhaps our work will help to eliminate at least some of these failures. It would undoubtedly have a positive impact not only on the condition of patients' teeth, but also on the confidence level of future dentists [4].

\section{CONCLUSIONS}

Students who complete the preclinical endodontics course are not adequately prepared for clinical practice. Endodontic treatment performed clinically by students during the third-year of their studies should be limited to single-rooted teeth with slight curvature. This procedure should be conducted under constant supervision of the experienced teaching staff.

However, the current model of education does not provide $1+1$ work. In most cases, one clinical teacher supervises several students; therefore in such situations, it is impossible for a single teacher to devote full attention to just one student. It seems that emphasizing the importance of preclinical education may have positive results in the future, which would manifest a higher treatment success rate and a lower incidence of complications.

In order to provide better dental education, further studies are planned on both hand instrumentation and NiTi rotary methods. We hope that this analysis will provide a foundation for the improvement of preclinical endodontics course and will enable better preparation of students for clinical practice.

\section{CONFLICT OF INTERESTS}

The authors declare no potential conflicts of interest with respect to the research, authorship, and/or publication of this article.

\section{References}

1. Abu-Tahun I, Al-Rabab'ah MA, Hammad M, Khraisat A. Technical quality of root canal treatment of posterior teeth after rotary or hand preparation by fifth year undergraduate students, The University of Jordan. Aust Endod J 2014; 40: 123-130.

2. Al-Omari MA, Dummer PM. Canal blockage and debris extrusion with eight preparation techniques. J Endod 1995; 21: 154-158.

3. Al Rahabi MK. Evaluation of complications of root canal treatment performed by undergraduate dental students. Libyan J Med 2017; 12: 1345582

4. Baaij A, Özok AR, Væth M, Musaeus P, Kirkevang LL. Self-efficacy of undergraduate dental students in endodontics within Aarhus and Amsterdam. Int Endod J 2020; 53: 276-284.

5. Berutti E, Negro AR, Lendini M, Pascualini D. Influence of manual preflaring and torque on the failure rate of ProTaper rotary instruments. J Endod 2004; 30: 228-230.

6. Eleftheriadis GI, Lambrianidis TP. Technical quality of root canal treatment and detection of iatrogenic errors in an undergraduate dental clinic. Int Endod J 2005; 38: 725-734.

7. Estrela C, Bueno MR, Barletta FB, et al. Identification of apical and cervical curvature radius of human molars. Braz Dent J 2015; 26 351-356.

8. Gluskin AH, Brown DC, Buchanan LS. A reconstructed computerized tomographic comparison of $\mathrm{Ni}$-Ti rotary GT files versus 
traditional instruments in canals shaped by novice operators. Int Endod J 2001; 34: 476-484.

9. Gonzalez Sanches JA, Duran-Sindreu F, de Noe S, Mercade M, Roig M. Centring ability and apical transportation after overinstrumentation with ProTaper Universal and ProFile Vortex. Int Endod J 2012; 45: 542-551.

10. Hülsmann M. Prevention and management of difficulties in endodontic treatment - problematic approach to endodontic treatment: part 1. Endodoncja.pl 2017; 3: 6-19 [In Polish].

11. Jafarzadeh $\mathrm{H}$, Abbott PV. Ledge formation: review of a great challenge in endodontics. J Endod 2007; 33: 1155-1162.

12. Kfir A, Rosenberg E, Zuckerman O, Tamse A, Fuss Z. Comparison of procedural errors resulting during root canal preparations completed by junior dental students in patients using an '8-step method' versus 'serial step-back technique'. Int Endod J 2003; 36: 49-53.

13. Khabbaz MG, Protogerou E, Douka E. Radiographic quality of root fillings performed by undergraduate students. Int Endod J 2010; 43: 499-508.

14. Lambrianidis T. Ledging and blockage of root canals during canal preparation: causes, recognition, prevention, management and outcomes. Endod Top 2009; 15: 56-74.

15. McGuigan MB, Louca C, Duncan HF. Endodontic instrument fracture: causes and prevention. Br Dent J 2013; 214: 341-348.

16. Moussa-Badran S, Roy B, Bessart du Parc AS, Bruyant M, Lefevre B, Maurin JC. Technical quality of root fillings performed by dental students at the dental teaching centre in Reims, France. Int Endod J 2008; 41: 679-684.

17. Peru M, Peru C, Mannocci F, Sherriff M, Buchanan LS, Pitt Ford TR. Hand and nickel-titanium root canal instrumentation performed by dental students: a micro-computed tomographic study. Eur J Dent Educ 2006; 10: 52-59.

18. Puri N, Chadha R, Kumar P, Puri K. An in vitro comparison of root canal length determination by DentaPort ZX and iPex apex locators. J Conserv Dent 2013; 16: 555-558.

19. Ribeiro DM, Réus JC, Felippe WT, et al. Technical quality of root canal treatment performed by undergraduate students using hand instrumentation: a meta-analysis. Int Endod J 2018; 51: 269-283.

20. Schäfer E, Dammaschke T. Development and sequelae of canal transportation. Endod Top 2006; 15: 75-90.

21. Schäfer E, Diez C, Hoppe W, Tepel J. Roentgenographic investigation of frequency and degree of canal curvatures in human permanent teeth. J Endod 2002; 28: 211-216.

22. Schäfer E, Lohmann D. Efficiency of rotary nickel-titanium FlexMaster instruments compared with stainless steel hand K-Flexofile - Part 1. Shaping ability in simulated curved canals. Int Endod J 2002; 35: 505-513.

23. Sonntag D, Delshen S, Stachniss V. Root canal shaping with manual and rotary Ni-Ti files performed by students. Int Endod J 2003; 36 : 715-723.

24. Ünal GÇ, Maden M, Orhan EO, Sarıtekin E, Teke A. Root canal shaping using rotary nickel-titanium files in preclinical dental education in Turkey. J Dent Educ 2012; 76: 509-513.

25. You SY, Kim HC, Bae KS, Baek SH, Kum KY, Lee W. Shaping ability of reciprocating motion in curved root canals: a comparative study with micro-computed tomography. J Endod 2011; 7: 1296-1300. 